\title{
¿Son los salarios reales o el nivel de demanda efectiva el principal determinante del nivel general de empleo? La teoría neoclásica vrs la teoría keynesiana
}

\author{
Ralael Pleitez
}

\section{Introducción}

En el campo de la Economia del Trabajo predomina la visión clásica con el análisis microeconómico del mercado de trabajo.

Una ldea central, dentro de este campo, es que un incremento en el nivel de salario nominal que conlleve a un nivel de salario real por encima del nivel de equilibrio causara una caida en la demanda de trabajo (acompanado de un aumento en la olerta de trabajo), creándose asi un nivel de desempleo mayor al de la lasa nalural de desempleo.' La anlerior relación se debe a dos efectos: (a) Efecto-producción que consiste en el hecho que el incremento salarial significa un incremento de coslos y de ahl un incremento de precios por lo cual la cantidad demandada por el producto se reducirá en alguna medida, y esto llevara a demandar una cantidad menor de trabajo, y (b) Electo-sustitución el cual consiste en el hecho que si el precio del trabajo se incrementa en relación con el precio del capilal, eslo tendera a una subslitución de trabajo por capilal. Asl esta teorla considera que el factor clave que explica el nivel de empleo es el salario real.

Keynes criticó el enloque clásico-microeconómico como un medio no adecuado para explicar el comportamiento del nivel agregado de empleo. Así el sugiere que el determinante principal del empleo agregado es el nivel de demanda agregada efectiva a través de la determinación del 


\section{nivel del producto.}

Las consecuencias a nivel de politica economica han sido que a partir de la finalización de la II Guerra Mundial hasta finales de los 70's predomino el enfoque Keynesiano con una politica monetaria y liscal expansiva que buscaba apoyar al nivel de demanda electiva y asl asegurar lasas de pleno empleo en las economias. El Keynesianismo ortodoxo entra en crisis a tinales de los 70's entre otros factores por la crisis en los niveles de déficit fiscales y de inilación en las economlas. En los 80's resurge el pensamiento clásico como dominante y con ello se privilegia el análisis económico desde el lado de la olerta. Asl se considera que es el salario real el principal determinante del empleo, criticándose la idea que bajo un manejo de la demanda agregada pueda asegurarse el pleno empleo. Es esta controversia la que ha motivado el presente trabajo.

Asl, primero se expondrán las ideas básicas sobre el mercado de trabajo expuestas por la leorla Neoclásica y Keynesiana. Segundo, se reportan los resultados de diversos trabajos hechos en la economia chilena sobre el cálculo de la elasticidad salario real-empleo. Las principales conclusiones son que la elaslicidad calculada con modelos neoclásicos muestran valores entre 0 y -0.5 , es decir, bastante inelásticos y el valor de dicha elasticidad calculada a partir de modelos macroeconómicos es contingenle al marco macroeconómico dominanle en la economía. (Donde juega un rol importante la exislencia o no de escasez de divisas y/o capilal, o bien la existencia de capacidad ociosa y restricción de demanda en las empresas). Tercero, se analizan las principales melodologlas que se han desarrollado para probar la causalidad de los salarios reales sobre el empleo y del producto sobre el empleo.

Dados los problemas de inconsislencia en los resultados oblenidos en diversas investigaciones, no se puede ofrecer una conclusión definitiva al problema planteado. Sin embargo, comienza a delinearse tres tendencias importantes: (a) Es difícil soslener empiricamente una relación inversa eslable enlre salarios reales y nivel de empleo. (b) la relación producto-empleo tiende a presentarse mas estable, y (c) los salarios reales parecen ser independientes del nivel del producto o estar positivamenle correlacionados, lo cual sugiere que el electo producto no opera, a nivel macro, en la forma indicada por la teoria Neoclásica. Asl, hasta el momenlo no se ha encontrado una evidencia empirica consistente que pruebe definitivamente la hipollesis Neoclásica o Keynesiana sobre el principal determinante del nivel de empleo. Lo cual, en principio, deberia prevenirnos de adoptar posiciones muy ortodoxas con una $u$ otra escuela. 


\section{A.1 Teorla Neoclásica.}

Esta teorla llene una visión optimista sobre el luncionamiento de las fuerzas del mercado (oferta y demanda), para el logro del pleno empleo en la economla. Se supone que con la acción de agentes individuales racionales que buscan maximizar sus funciones de beneficio, sujeta a la restricción de recursos escazos, el mercado asegurara el pleno empleo. siempre y cuando se respeten los precios relativos que se deriven de la libre interacción entre oferta y demanda. De lal forma, que si los precios son plenamente flexibles, puede asegurarse que el mercado tiene la capacidad de reajustarse a si mismo ante cualquier shock que pueda sutrir la economia. En este sentido, la exislencla de una tasa de desempleo superior a la tasa de desempleo nalural, se deberá a la falta de flexibilidad de los precios impuesta por algúnos agente/s de la economia, que eslará obstaculizando el auto-ajuste del mercado por medio de la olerta y la demanda que reaccionan según las sefiales del mercado: los precios relativos. En general, esta teoría se caracteriza por un análisis microeconómico en el cual se privilegia las consideraciones derivadas del lado de la oferta.

En concreto, el modelo Neoclásico sobre el mercado de trabajo consiste en lo siguiente:

(a) Se parte de condiciones ideales en las cuales los salarios y precios son plenamente flexibles, y las empresas esperan que toda su producción será vendida al nivel de precios prevalecientes, en el marco de un mercado competitivo. Asimismo, se asume, que en el corto plazo el único factor de producción variable es el trabajo.

(b) La demanda de trabajo es una función derivada de la función de producción. La función de producción mostrara rendimientos marginales decrecientes de parte del factor trabajo. Esto se debe al hecho de que el stock de capilal es tijo en el corto plazo y que por lo lanto el incremento en el producto provocado por el empleo de una unidad mas de trabajo disminuirá mientras la cantidad de trabajo utilizado aumenla. La demanda de trabajo es igual a la función del producto marginal del trabajo para distintos niveles de unidades de trabajo. Esta función se caracleriza por una pendiente negativa que refleja los rendimienlos decrecientes del trabajo. Asimismo, es importanle resaltar que cada empresa tendrá una demanda de trabajo diferente en correspondencia con la relación técnica trabajo-capital que posea en su función de producción. La función de demanda agregada de trabajo para un sector de la economia o de la economía en su con- 
junto se derivara de la sumatoria de las funciones de demanda de trabajo de cada empresa. La función de demanda de trabajo contestara la pregunta sobre cuantos trabajadores esta dispuesta a contralar una empresa. La teorla dirá que el optimo de conlratación será en el punto en el cual el salario real se iguale al producto marginal del trabajo. En otras palabras, en el punto en el cual el valor de la cantidad producida por el ultimo trabajador contratado se iguale al salario nominal pagado por la empresa. En esta lorma queda establecida una relación inversa y estable entre salarios reales y la cantidad demandada de trabajo.

(c) La oferta de trabajo estará dada por la declsión que tomen los trabajadores en la elección entre horas de trabajo y horas de acio. De tal forma que la oferla de trabajo tenderá a aumentar en la medida que los salarios reales aumentan, en tanto que estos mayores salarios compensaran las horas de ocio que el trabajador sacrifica por aumentar las horas de trabajo, este es el llamado electo suslitución y el cual es positivo. Por otra parte, existe el electo ingreso, el cual tiene una relación negativa con las horas de trabajo que una persona ofrece en el mercado, ya que si el ingreso de una persona aumenta su disposición para trabajar mas horas disminuirá. Es importanle, resaltar que en loda variación de salarios se dan los dos efectos al mismo tiempo y actúan en sentido opuesto, dependerá en cada caso cual efecto domine para poder determinar el cambio en la olerta de trabajo inducido por un cambio de salarios. ${ }^{2}$

(d) Es la Interacción de la oferla y la demanda de trabajo la que determinara en cada sector de la economía el nivel de salario real que asegurara el pleno empleo. Dado esle nivel de salario real cada empresa determinara el numero de trabajadores que contratara y luego este nivel de empleo a través de la función de producción determinara el nivel de producción.

En sínlesis, de acuerdo con la teoría Neoclásica es el nivel de salario real (determinado en el mercado de trabajo), el que determinara el nivel de empleo y esle a su vez al nivel de producción.

Una acolación importante dentro de este modelo es la siguiente: el manejo de políticas que alectan la demanda agregada no afectan en el largo plazo el nivel de equilibrio alcanzado por el mercado. Por ejemplo, si el Banco Central incrementa la cantidad de dinero al nivel de precios prevalecienles, la olerla real de dinero será mayor e inducirá a un menor nivel de la tasa de interés, lo cual a su vez implicara un incremento en la demanda de bienes. Esle incremento en la demanda provocara un incremento en el nivel de precios lo cual provocara en el corto plazo una 
reducción en el nivel de salarios reales. Esto inducirá a un Incrememo en la demanda de trabajo y una reducción en la oferta cuyo electo neto será un incremento en el nivel nominal de salarios hasta el pumto de Igualar al nivel de salarios reales previos al incremento en la oferta de dinero, con lo cual se estará en el mismo nivel de pleno empleo. Asl, el dinero es neutral dentro de este modelo, to cual significa que no es capaz de afectar las variables reales de la economia en el largo plazo. (Dornbusch, 1987, p.464).

\section{B. Teorla Keyneslana.}

Esta teorla considera que existen deflcienclas en los mecanismos de ajuste por parte del mercado, y por tanto el gobiemo puede a través del manejo de la demanda agregada ayudar a la regulación de las economias capitalistas. En este sentido, Keynes expresó que no se puede creer que una politica de salarios flexibles pueda ser capaz de mantener un estado contínuo de pleno empleo (Keynes, 1936,p.267).

Esta teoría enfatiza los movimientos en la demanda agregada como generadores de fluctuaciones en los niveles de producción y empleo de las economlas. Asimismo, considera que un cambio en la confianza sobre el desenvolvimlento de la economla o en otras palabras un cambio en las expectativas de parte de los empresarios y/o consumidores puede ateclar el rivel de demanda agregada para bienes y por b tarto cambios en los niveles de producción y empleo de la economía a nivel glbbal.

Especificamente, la teorla Keynesiana sobre el mercado de trabajo consisle en lo siguiente: (a) En cuanto a la oferta de trabajo, Keynes dice que esta no es función de los salarios reales como una sola variable, y si esto es asl la teoría clásica del mercado de trabajo queda indelinida (Keynes, 1936,p.8). Para Keynes la oterta de trabajo es función de tres variables: el nivel de salarios nominales, el nivel de precios y el nivel de salarios reales. En este sentido, Keynes critica la idea de los clásicos sobre que el cambio del análisis en el mercado de trabajo en variables reales o nominales es neutro para las conclusiones que de ahl se derivan. De tal manera, que puede darse el caso que ante un incremento de los salarios reales, la oferta de trabajo disminuya si es acompanado por una calda en el nivel de salarios nominales (Drobny, 1988,p.93).

Keynes apoya su posición con dos principales argumentos: (1) La hipólesis de los salarios relativos, con base en la cual, argumenta que aquellos trabajadores que acepten una reducción en los salarios nominales en relación con otros trabajadores, sulrirán una reducción relatlva en sus salarios reales, y esto constiluye una justificación suficiente para 
que to resislan (Keynes,1936,p.14). (2) Argumenta que el nivel de salario real no es determinado dentro del mercado de trabajo. Es decir, los trabajadores y los empresarios en sus negociaciones sobre salarios no pueden decidir más que sobre el nivel de salarios nominales. El nivel de salario real es determinado por la fijación de un determinado nivel agregado de precios. Asl Keynes concluye que el nivel de empleo es determinado en el mercado de bienes en el punto de equilibrio entre la oferta y la demanda de bienes. Específicamente dice: 'La propensión marginal a consumir y la lasa de inversión determinan entre ellas el volumen de empleo, y el nivel de empleo esta únicamente relacionado con un determinado nivel de salarios reales y no a la inversa" (Keynes,1936, p.30).

La anterior afirmación no significa que Keynes no acepte el clásico vinculo entre producto marginal del trabajo y el nivel de salarios reales. El dice en el capitulo 2: "...con una delerminada organización, equipo y técnica, los salarios reales y el nivel de producto (y de ahi el nivel de empleo) están únicamente correlacionados, de ahl que, en general, un incremento en el empleo puede ocurrir solo con el acompanamiento de una reducción en el nivel de salarios reales. Así yo no disputo este hecho vilal que los economislas clásicos acerlaron correctamente" (Keynes,1936,p.17). Sin embargo, Keynes crilica la función de demanda de trabajo clásica con los siguientes puntos: (1) La demanda del producto de la firma no entra directamente dentro de la lunción de demanda real de trabajo. (2) Keynes es claro en decir que el efecto directo de cambios en lo salarios nominales sobre los costos de producción, y de ahi al nivel de producto y empleo no opera como es sugerido por la teorla clásica (Drobny,1988, p.98). El acepta que este mecanismo opera para una determinada empresa, pero no puede trasladarse el argumenio para todo un sector de la economla, a menos que también se transtiera el supuesto que la demanda electiva agregada esta lija. En sus propias palabras: "..nadie desearía negar la proposición que una reducción en los salarios nominales acompanados por la misma demanda efectiva agregada anterior estará asociado con un incremento en el nivel de empleo, la pregunta precisa en este problema es si la reducción en salarios nominales irá o no acompanada por el mismo nivel de demanda efectiva agregada" (Keynes,1936,p.259). En otras palabras, no se puede ignorar el efecto ingreso que a nivel agregado tiene un cambio en los salarios nominales.

Keynes analizó una variedad de posibles efectos que una caída en los salarios nominales y reales pueden tener sobre la demanda efectiva y de ahl sobre el nivel de producción y empleo global de la economia. El primer efeclo que estudió fue el efecto distribución. El dice que una reducción en los salarios nominales tendera a reducir los precios en 
algún nivel (p.262). Si esto es asl, se tendrla una redistribución del ingreso real desde los asalariados hacia olros tactores de producción cuya remuneración no se haya reducido, y de los capitalistas a los rentistas quienes tienen el Ingreso tijo en términos nominales. Dado que la propensión marginal a consumir de los asalariados es mayor que la de los capitalistas y renlistas, los efectos de dislribución disculidos arriba tenderán a disminuir la propensión marginal a consumir y de ahl el nivel de demanda agregada efectiva. Por lo tanto, existirá un efecto negativo para el nivel de empleo en el corto plazo.

De ahi que Keynes concluya lo siguiente: "nuestra esperanza de resultados lavorables en el empleo deben basarse principalmente en un incremento en la inversión ya sea a través de un incremento en la eliciencia marginal del capital o de una reducción en la tasa de interés" (p.265). . Asi Keynes analiza el efecto de la caida en los salarios nominales sobre la eficiencia marginal del capital. Por una parte, ésta tendera a incrementarse dado que si los salarios nominales se reducen más que los precios los benelicios por unidad tenderán a incrementarse, ceteris paribus. Por otra parte, la eliciencia marginal del capilal lenderá a reducirse dada la calda en el nivel de precios y si la expectativa es que continué, los beneficios futuros tenderán a reducirse. Asimismo, la demanda del producto a nivel agregado puede caer dada la disminución en la propensión marginal a consumir (producido por el efecto distribución), con lo cual los beneflcios totales pueden disminuir. En resumen, el electo neto sobre la eficiencia marginal del capital es ambiguo. En cuanto a la tasa de interés, dirá que dada la reducción en los salarios nominales y preclos provocaran una caída en la demanda de dinero y por tanlo una reducción en la lasa de interés. 4 Lo cual tendrá un efecto positivo sobre el empleo si la demanda de inversión es razonablemente elástica a la lasa de inlerés.

En resumen, Keynes argumenla que en una economía cerrada los electos sobre el empleo de una reducción/aumento en los salarios nominales dependerán del peso relativo de cada uno de los electos analizados. Cabe resaltar que el punto crucial en el resullado neto de los efectos depende del comporlamiento de la lunción inversión, la cual es volálil, ya que se encuentra dominada por las expectativas y conjeluras de los empresarios.

En conclusión, la teoría Keynesiana sugiere que un incremento en el nivel real de gasto produce un incremento en el nivel de producto $y$ empleo global de la economía, cuando las empresas presentan una resIricción de demanda. En esle marco, puede suceder que un incremento en los niveles de salarios reales produzcan mayores niveles de deman- 
da, producto y empleo o que una disminución en los salario reales produzcan una reducción en la demanda, producto y empleo. En una trase, en el modelo Keynesiano no se requiere necesariamente una reducción en los salarios reales para oblener un mayor nivel de empleo global. La relación fundamental en este modelo es entre la demanda efectiva que determina el nivel de producto y este el nivel de empleo. Los salarios tendrán efectos sobre el nivel de empleo, pero solo a través de los electos que estos produzcan sobre el nivel de demanda electiva real y por el efecto sustilución por capilal, cuando éste deja de ser tijo. Por ultimo, dentro de este modelo la política fiscal y monetaria tienen un papel importante a cumplir para estimular la demanda efectiva y con ello el nivel de producto y empleo. Especialmenle se aconsejaría una política que permiliese mejorar las expectalivas de los capitalislas y en general la contianza de parte de los consumidores en la economia. De ahi que, por ejemplo la inversión pública puede ayudar a que la inversión nacional lenga un comportamiento más estable, una politica tributaria con efectos redistributivos a los sectores que poseen una mayor propensión marginal a consumir puede mejorar el nivel de consumo real, de demanda electiva y de ahl mejorar las expectalivas de venta de las empresas y asl estimular la inversión privada, logrando con todo eslo importantes electos favorables sobre el nivel de producto y empleo general de las economias capitalistas.

\section{B. Revisión de Estudlos Empiricos.}

\section{B.1 Elasticidades salarlos reales-empleo y empleo-producto en la economia de Chlle.}

Dentro de los esludios econométricos para calcular la elasticidad salarios reales-empleo se distinguen dos grandes grupos: (a) estudios de equilibrio parcial o sectorial, que utilizan la demanda de trabajo de la escuela clásica y (b) análisis macroeconómicos.

Las caracteríslicas principales en los modelos de equilibrio parcial son las siguientes: (a) el enfoque leórico comun es la estimación de "demandas de trabajo neoclásicas", en las que se supone un proceso de ajuste parcial to que permite distinguir elasticidades de corto y largo plazo, (b) se basan en datos trimestrales para el periodo posterior a 1974, y (c) ulilizan inlormación del sector manufacturero (Solimano, 1983; Riveros y Arrau,1984), pero exislen estudios para el empleo agregado (Rojas, 1987).

Los principales resultados de los modelos de equilibrio parcial son: una elasticidad salarios reales-empleo de corto plazo igual a -0.35 y de 
largo plazo menores a la unidad. En algunos casos este parámetro se ha encontrado estadísticamente no significalivo. En cuanto, a la elasticidad empleo-producto de corto plazo en promedio han encontrado un valor de 0.5 y menor a la unidad en el largo plazo. Generalmente, este parámetro se ha enconlrado esladisticamenle significativo.

En los análisis macroeconómicos el signo y el valor de la elasticidád salarios reales-empleo depende de la especilicación del modelo.

En los modelos de Restricción Extema dominante en los cuales se suponen que las reservas internacionales netas son fijas, un aumento de los salarios reales conlleva a una apreclación del tipo de cambio real, lo cual desestimula las exportaciones y eslimula las importaciones por lo que el efecto nelo es un deterioro de la balanza comercial. Para compensar esta siluación, se tendrá que reducir el gasto interno con lo cual se afecta negativamenle el nivel de empleo. En esle marco, la elasticidad salarios reales-empleo es negativa y su magnitud dependerá de: la elasticidad salarios nominales-nivel general de precios, la elasticidad exportaciones-tipo de cambio real, la elasticidad producto-exportaciones, y la elasticidad empleo-producto. En un estudio hecho por Cortázar (1983), enconiró una elasticidad salarios reales-empleo de corto plazo igual a -0.526 . Meller y Solimano (1987) encontraron un valor de -0.34 para la misma elasticidad. La diferencia entre estos resultados se debe a que Cortázar uso una elasticidad empleo-producto de 1.0 y en el otro estudio dicha elasticidad tue de 0.6 .

Solimano (1986) examinó dicha elasticidad bajo tres regímenes macroeconómicos distintos: (a) Keynesiano, es decir, con restricción de demanda efectiva y efectos consumo privado (efecto distribución) y público dominantes, 10 cual implica que un incremento de los salarios reales pueden inducir a incremento en el nivel de producto y empleo. Asl, encontró una elasticidad de corto plazo igual a 0.45 . (b) Clásico, es decir, con restricción de capital, la elasticidad fue de -0.34 .

En conclusión, estos estudios muestran que la elasticidad empleosalarios reales es contingente al régimen macroeconómico dominante en un momento delerminado en la economia. Asl en un marco de resIricción de divisas y/o escasez de capital, la elaslicidad de corto plazo estará entre 0 y -0.5. La elasticidad empleo-salarios reales puede ser positiva si existe capacidad ociosa y/o suficiente disponibilidad de divisas. Sin embargo, en general siempre vamos a tener un problema de agregación, es decir, en realidad al mismo tiempo en la economia vamos a encontrar sectores con problemas de restricción en la demanda de sus productos y otros sectores restringidos por capacidad productiva y/o disponibilidad de divisas. 


\section{B.2 Anallsio de Causalldad.}

Es conocido que los analists de regresion entre varlables, que han sido especticadas en ecuaclones, colo nos cicen si exlste o no una asoclación entre dichas variables, pero no nos indlcan relactón de causalidad.

En 1969, Granger Imrodujo el uso de series de tiempo y análisis de regresión para realizar pruebas de causalldad entre varlables. La defintclón de causalidad que Granger ofrece (en el marco de estos especlftcos Instrumentos estadísticos) es la sigulente: Si la variable Xt "causa" a la variable $Y t$, entonces la predicción de Yt será mejorada cuando valores pasados de Xt son anadidos. Más formalmente, sl tenemos el stguiente modelo:

$$
\begin{aligned}
& Y t=a j Y t-j+U t \\
& Y t=a j Y t-j+b j X t-j+E t
\end{aligned}
$$

Donde Ut, Et son los errores de estimación y a, b son los coeficienles de regresión.

Entonces si XI causa a Yt implica que la varlanza del error de predicclón para la variable $\mathrm{Yt}$ en la ecuación 2 debe ser significativamente menor que la varianza derivada de la ecuación 1.

Ora importante detinición es la de retroalimentación. Esta sucede cuando se encuentra que Xt causa a Yt y también Yt causa a Xt (cuando la variable Xt pasa a ser la variable dependiente en las ecuaciones 1 y 2).

Drobny realiza un interesante análisis sobre tres principales supuestos en los que descansa este tipo de prueba de causalidad-Granger:

1. Este método asume que el futuro no puede causar el pasado, en otras palabras este método asume que existe una correspondencia directa entre orden causal y orden temporal (Granger, 1969, p.428). Es decir, si se encuentra que los movimientos en XI generalmente preceden los de Yt, enlonces la proposición que XI causa a YI no puede ser rechazada. Esta ultima afirmación es polémica. Inclusive se han encontrado ejemplos en donde el orden causal y temporal han sido invertidos.

Drobny examina dos ejemplos interesamles y pertinentes a este pun10. Primero, un modelo deterministico usado por Tobin en 1970, para argumentar que aunque los picos en la oferta monelaria parecen conducir a los picos del ingreso nominal en los US, esto no implica que cambios en la oterla monetaria causen cambios en el ingreso nominal (Drobny, 1988, p.151-154). Este ejemplo (puede verse en el trabajo de Tobin y el análisis que Drobny hace de éste), nos deja dos lecciones: (a) 
Demuestra los peligros de aceptar la evidencla de secuencla de liempo como prueba emplíca de causalidad (Tobin, 1970, p.303), (b) Tobin muestra corno en un modelo delerministico la correspondencla entre orden temporal y causal, no es condicion necesarla de causalidad, de tal manera que las pruebas de causalidad requleren de modelos estocásticos. Segundo, aun en modelos estocasticos se pueden encontrar ejermplos en los cuales el orden temporal y causal se puede invertir por la influencla de variables omitidas. Por ejemplo, Nelson y Schwert (1977) realizaron una prueba de causalidad en la que encontraron que la varianza de los errores de predicción de la inflación era significativamente reducida cuando la tasa de interés se Inclula en su predicclón. También encontraron que el coeficiente de la tasa de Interés era signilicalivamente dilerente a cero. En la estimación del modelo la tasa de interés era definida para el inicio del periodo y la tasa de inflación era delinida como la lasa de cambio en los precios ocurridos durante dicho periodo, asl los movimientos de la tasa de interés precedian a la tasa de inflación. Con loda esta evidencia se podla concluir que la lasa de interés causaba a la lasa de inflación. Sin embargo, como apunta Drobny esla conclusión puede ser enganosa ya que la fijación de la tasa de interés nominal puede ajustarse a los cambios esperados de la tasa de inilación. El punto clave acá es como se forma esta expectaliva de la tasa de inflación esperada. Un posible escenario serla asumir que la lasa de crecimiento de la oferta monelaria "causa" a la lasa de inflación, si esto es asi la lasa de inflación esperada eslaría delerminada por la lasa esperada de crecimiento en la oferta monetaria. Por ende, la tasa de interés al principio del periodo puede tomar en cuenta los cambios de política monelaria y su incidencia en la oferta monetaria para formarse la expectativa de la tasa de inflación futura y asl ajuslarse a ésta. En este sentido, en realidad la tasa de inflación esperada estaría determinando a la tasa de interés y no a la inversa. El resultado empírico encontrado puede ser engañosa por la ausencia de una variable importanle en el análisis, la tasa de crecimiento de la oferta monetaria. En definitiva, de acuerdo con Drobny, este principio de correspondencia entre orden temporal y causal permite el uso de la metodologia desarrollada por Granger como una prueba de condición necesaria pero no suficiente para la existencia de causalidad entre variables.

2. Mayoritariamente este tipo de modelo es de carácter bivariable, asumiendo que cualquier variable adicional es independienle de Xt y $\mathrm{Yt}$, y que su influencia sobre la variable dependiente es en una forma no sistemática. (Drobny, 1988, p.150, 158-159). Obviamente este supueslo limita el uso de esta metodología para un conjunto de pregunlas en economila que implican el uso de variables que son determinadas en 
modelos de carácter multivariable.

3. La definición de causalidad-Granger es en relación sólo con variables estocásticas y estacionarias. Entendiendo por una variable eslacionaria a aquella variable que posee una media y varianza constante, las cuales son independientes del liempo (Anderson, 1971, Ch.7). Drobny senala tres razones por las cuales es importante la estacionalidad de las variables: (a) Para poder generalizar la conclusión a través del tiempo, (b) nos permite aproximarnos a una situación de experimento controlado, ya que si tenemos un palrón de conducta consistente en cada variable, podremos decir que innovaciones en $X t$ son usualmemte seguldas por similar tipo de shock en $Y t$ y de ahl que se pueda inferir que $X t$ causa a $Y t, y$ (c) dado que Irecuentemente las variables macroeconómicas sufren de colinealidad (inducidas por la influencia de variables externas que no se incluyen en el modelo), con una variable estacionaria se sulre menos de este problema.

De tal forma, que para una apropiada aplicación de la metodologia de Granger se requiere que las variables $X_{1}$ y $Y_{1}$ sean Iranslormadas a variables estacionarias. Drobny propone el siguiente mélodo para esto: si se tratan de transformaciones lineales se tomaría la primera o segunda diferencia de la variable en una serie de tiempo, realizando la regresión de dicha serie. Los residuos oblenidos por dicha operación serlan usados como las nuevas variables eslacionarias $x t$, yt . A este proceso se le denomina "Yiltrar" una variable. En cierto casos, será necesario adicionalmente "blanquear" a la variable, es decir, eliminar cualquier problema de aulocorrelación en una variable estacionaria. Esle punto es relevante en el siguiente aspecto, las técnicas empiricas disefíadas para probar causalidad difieren en la forma de filtrar y/o blanquear a las variables y eslo puede ser un factor que explique el porque de resultados conflictivos entre diversos estudios.

Dentro de las diferentes investigaciones de causalidad entre salarios reales y empleo, han habido tres principales procedimienlos empíricos para realizar las pruebas de causalidad-Granger:

1. La prueba directa Granger. El sistema de regresiones es el siguiente:

$$
\begin{aligned}
& x t=a j x t-j+b j y t-j+e 11 \\
& y t=c j y t-j+d j x t-j+e 2 t
\end{aligned}
$$

Donde, $x t e y t$ son variables estacionarias transformadas a partir de las variables originales $X t \in Y t .(a, b, c, d)$ son coeficientes de regresión y e1, e2 errores de estimación. Este mélodo ocupa lantos rezagos como 
sean posbles probar, o en olras palabras se pueden eliminar aquellos rezago6 cuyos coelicientes comiencen a ser de un tamano insignificante. Esta prueba requiere el cálculo del F-estadistico del grupo de rezagos significativos de la variable explicatoria. Si este es significativo al nivel del $10 \%$, entonces dicho resultado sugiere que la variable explicatoria causa en el sentido Granger a la variable dependiente. Drobny sugiere también calcular la suma de los coelicientes de los rezagos de la variable explicatoria estimar su significancia estadistica para poder ver la permanencia y dirección del electo de la variable explicatoria sobre la variable dependiente. Este método puede extenderse a un sistema mullivariable a Iravés de la introducción de adicionales variables explicatorias, lo cual es una ventaja del mélodo. Dos defectos son que sus resultados se ven influenciados por el numero de rezagos y el tamaño muestral con el que se trabaje, con lo cual no se puede distinguir entre causalidad temporal o permanente.

\section{La prueba de doble entrada: Sims.}

El modelo de regresiones que se utiliza es el siguiente:

$$
\begin{aligned}
& x t=\text { aj } y t-j+u 1 t \\
& y t=b j x t-j+u 2 t
\end{aligned}
$$

Donde, $x t$ e yt son variables estacionarias, $(a, b)$ son coelicientes de regresión y (u1, u2) errores de eslimación. Esta prueba se basa en valores pasados y futuros de $x, y$. Si $x$ causa a $y$, entonces los valores futuros de $x$ en la ecuación 6 deben se insignificalivamente diferente de cero y los valores futuros de y en la ecuación 5 deben ser significalivos como grupo. Esle método requiere el calculo del F-estadístico del conjunto de valores futuros de las variables. Si el F-esladístico es signilicativo al nivel del $10 \%$ implicara que la variable dependiente causa (en el sentido Granger) a la variable explicatoria.

3. La prueba de correlación cruzada: "S-estadístico", desarrollada por Pierce y Haugh.

Ante todo, es importante senalar las diferencias que existen entre esta melodologla y la prueba Granger: “(a) Es una prueba de independencia mas que de causalidad; (b) está basada en un análisis de correlación cruzada más que un análisis de regresión; y (c) cada variable tiene un fittro diterenle, el cual es determinado emplricamente" (Drobny, 1988, p.165).

Asi el primer paso seria delerminar los filtros mas apropiados para las variables Xt, YI con el objelo de producir series "blanqueadas" Xt, yt. ${ }^{5}$ El segundo paso seria calcular los coeficientes de correlación cruza- 
da para cada rezago utilizado en las variables "blanqueadas" calculadas previamente.

Haugh (1976) mostró que en el caso especlal de Independencia la suma de los coeficientes de correlaclón al cuadrado multiplicado por el tamaño de la muestra produce un estadístico "S" el cual está distribuido en la forma de una distribución chi-cuadrado.

$$
S=n \quad r^{\wedge}(k)
$$

Donde, $m^{\wedge}(k)$ son las correlaciones cruzadas estimadas y " $n$ " el tamaho de la muestra.

El problema con esta prueba es que cuando las variables están causalmente relacionadas la disıribución S-esladístico no esta bien definida (Drobny,1988,p.167). (Pierce y Haugh, 1977, p.284). Lo cual puede conllevar a que esta prueba no rechaze la independencia entre variables cuando en realidad existe una relación de causalidad entre ellas. (Error tipo II en la prueba de hipótesis).

Revisión de la evidencla empirlca de la aplicación de las pruebas de causalldad.

El esludio empirico sobre la relación inversa entre empleo y salario real tiene una larga historia. Dunlop (1938) y Tarshis (1939) reportaron una correlación positiva entre salarios reales y salarios nominales, to cual le daba un movimiento pro-ciclico a los salarios nominales. Ruggles (1940) y Tobin (1948) criticaron la signilicancia estadistica de los resullados de Dunlop y Tarshis, 10 que motivo mas estudios. Kuh. (1966) y Bodkin (1969) no encontraron una relación signiticativa entre salarios reales y empleo. En los 70 's se realizaron nuevos esludios, los cuales anle lodo comenzaron crilicando la melodologla ocupada en los estudios anteriores, tal es el caso de Bodkin (1969) quien utilizó regresiones conlemporáneas (enconlrando una relación positiva entre salario real y empleo), ignorando el efecto de los rezagos. Los nuevos estudios utilizaron las pruebas de causalidad-Granger. En los 80 's se realizaron nuevas invesligaciones cuyos resultados entraron en conflicto con los principales resultados obtenidos en los 70's. Veamos en detalle el resultado de estos estudios:

Neltci (1978) utilizo "la prueba Sims". Los datos utilizados fueron los siguienles: el empleo, que lue definido como el numero de empleados en las planillas del sector manufacturero de los US. El salario real definido, como el sueldo promedio por hora (excluyendo las horas extras) deflactado por el indice de precios al consumidor. El periodo utilizado 
fue datos mensuales desde 1948 hasia 1971. Utilizo 12 adelantos (leads (+s)) mensuales y 24 rezagos (lag (-k)) como la variable del lado derecho en las ecuaciones. Los principales resullados fueron: (a) Para la relación salarios reales sobre el empleo el F-estadistico es 3.26, el cual es significativo a un nivel de signilicancia del $5 \%$. Lo que suglere que los salarios reales "causan" al nivel de empleo sin retroalimentación; (b) Encontró también que la suma de los coeflcientes de los salarios reales es negativa y significaliva cuando se utilizan 12 o más rezagos mensuales, y (c) Encontró que el coeficiente contemporáneo es positlvo y signiticalivo estadisticamente, con lo cual explicaba of resultado de Bodkn.

Sargent (1978) utilizó la prueba directa Granger. Ocupó datos trimestrales para US. en el periodo 1948(1)-1972(4) con 4 rezagos para cada variable. Como deflactor utilizo el indice de precios al consumldor. Los datos no tueron filtrados y fueron utilizados en forma logarímica. Los principales resultados fueron: (a) El F-estadistico para la prueba de cau. salidad de los salarios reales sobre el empleo fue signilicativo al nivel de signiticancia del $10 \%, y$ (b) ninguno de los coeficientes individuales de la variable causal fue estadisticamente significativo.

Kirkpatrick (1981) utilizó la prueba directa Granger. Reeslimo la regresión Sargent para un período mayor: 1948-1977. Usó el deflactor Implicito del sector manulacturero. El resultado principal es que el Fesladistico deja de ser significativo estadísticamente, con lo cual no se puede sugerir la causalidad de los salarios reales sobre el empleo.

Kirkpatrick (1982) utilizó una prueba directa-Granger en una versión multivarlable. Usó datos trimestrales para el sector manufacturero alemán durante 1960(1)-1979(4). Incluyó los precios de las materias primas utilizando al deflactor implicito del sector manulacturero. Los princlpales resultados fueron: (a) Para el periodo 1960(1)-1979(4), encontró un Festadlstico signiticalivo para un nivel de signiticancia del $17 \%$ para la relación salarios reales sobre el nivel de empleo; (b) para el periodo 1960(1)-1969(4), el F-estadistico es significativo a un nivel de signiflcancia del 30\%, y (c) para el periodo 1970(1)-1979(4), el F-estadistico es significativo al nivel de significancia del $5 \%$.

Geary y Kennan (1982), usó la prueba de correlación cruzada: Sestadistico y una prueba F-estadístico y regresión sugerida por Geweke (1980). ${ }^{\circ}$ Utilizaron dalos trimestrales para el sector manufacturero de 12 palses del OECD.' El deflactor uilizado lue el dellactor Implicito del sector manutacturero. (En 10 de los 12 paises, y el deflactor de toda la economia para los otros dos). El principal resultado fue: Con la prueba S-estadistico sólo Nueva Zelandia e Irlanda (con los datos diferenclados) rechazaron la hipótesis de independencia con un nivel de signili- 
cancia del 10\%. Para comparar su metodología con los resultados de Neftci y Sargent, los autores utilizaron sus pruebas pero para el período muestral 1948-1971, utilizando datos mensuales para el caso Neftci, trabajando con el índice de precios al consumidor tal como lo hicieron Neftci y Sargent, y para analizar el caso Sargent utilizaron el nivel de empleo de toda la economía tal como Sargent to hizo. Los principales resultados fueron: Las hipólesis de independencia fueron rechazadas con un nivel de signiticancia del $5 \%$. Y lograron reproducir los resultados de Neftci con una suma negativa y significativa estadísticamenle de los coeficientes de los salarios reales sobre el empleo. Por to cual, concluyeron que la diferencia original de resultados se debe al uso de un deflactor de precios y lamano muestral diferente mas que al uso de diferente técnica estadística.

Drobny (1988) utilizo las tres melodologías: la prueba directa Granger, la prueba Sims y la prueba de correlación cruzada (S-estadlstico). Trabajó con datos trimestrales para el sector de la manulactura en el Reino Unido durante el período 1957(1)-1981(2). El salario real lue determinado a partir del salario promedio nominal (excluyendo horas extras), el cual fue deflactado con el indice de precios implícito del sector manufaclurero. (También construyó olra serie de salarios reales usando el índice de precios implicilo para loda la economia). Tomó a todos los empleados en el sector manufacturero, como nivel de empleo. El precio real de las malerias primas lue delerminado tomando los precios de las materias primas compradas por la industria manufacturera deflactado por el Indice de precios implicito del sector. Los principales resultados fueron: Con la prueba Granger y Sims, para el periodo 1952 (1)-1981(2), se encontraron F-estadisticos significativos al nivel de signilicancia del $5 \%$ para la relación de los salarios reales sobre el empleo (a excepción de la prueba Granger con el deflactor para toda la economia, el cual fue significalivo al nivel de significancia del $10 \%$ ). Este resultado sugiere que los salarios reales "causan" al nivel de empleo sin retroalimentación (a excepción de la prueba Sims con el deflactor de toda la economia). Calculó el efeclo de largo plazo del cambio de salarios reales sobre el empleo, el cual es de -0.56 en la prueba Sims y 0.20 dentro de la prueba directa Granger. Con la prueba "S-esladlslico" se obtiene un resultado inconsistente con las otras dos pruebas. (Drobny, 1988, p.189).

Drobny calculo también la prueba Granger incluyendo el precio de las malerias primas durante lodo el periodo 1952(1)-1981(2). Se ulilizaron 4 rezagos para el empleo y las materias primas, y 10 rezagos para los salarios reales. Los resultados sugieren que los salarios reales y precios reales de las materias primas "causan" al nivel de empleo. Se encontró 
una elasticidad salarios reales-empleo de largo plazo igual a -0.76 . (Drobny,1988,p.195-199). Luego, recalculo las pruebas Granger y Sims para el periodo 1951(2)-1971(4) y los F-estadisticos dejaron de ser significalivos. Finalmente, realizó la prueba Granger con las tres varlables para el periodo 1951(2)-1971(4). En este caso el F-estadístico fue solo signiticativo para el precio real de las malerias primas.

Drobny (1988) realizo la prueba de causalidad del nivel del producto sobre el empleo. El aplicó primero una prueba Granger bivariable (con 4 rezagos para cada variable) para el período 1952(2)-1981(2) en el Reino Unido." En la relación, corriendo del nivel de producto hacia el empleo. se encontró un F-estadistico significativo al nivel de significancia del $5 \%$ . Sin embargo, se encontró también significativo la relación del empleo sobre el producto al mismo nivel de significancia, pero la suma de los coelicientes del empleo no resultaron estadisticamente significativos. Luego el autor recalculó las ecuaciones para el periodo 1957(2)-1971(4) y los F-estadisticos se mantuvieron signilicalivos. La significancia de la suma de los coeficientes del producto se manluvieron. El autor concluye que la relación que corre del producto hacia el empleo es estable, ya que logra mantenerse a pesar de los cambios en el periodo muesiral. (Drobny, 1988,p.212,223). Por ultimo, Drobny calculó un slstema multivariable (salarios reales con 10 rezagos, empleo y producto con 4) con la prueba directa Granger. Para el periodo 1957(2)-1981(2) el resultado iue el siguiente: Con base en los F-estadísticos se sugiere que sólo los salarios reales "causan" al nivel de empleo sin relroalimentación y solo el nivel de empleo "causa" al producto sin relroalimentación. Sin embargo, cuando se analizan las sumas de los coelicientes, tanto los del producto como los de los salarios reales, son signilicativos a un nivel de significancia del $10 \%$. Es interesante notar que con base en esta prueba los salarios reales "causan" al nivel de empleo, pero no al nivel de producto. Drobny realiza una úllima prueba con el mismo modelo pero para el perlodo 1957(2)-1971(4); el resultado lue: Con base en los F-estadisticos sugieren que el producto "causa" al nivel de empleo, los salarios dejan de ser "causantes" del nivel de empleo, y se mantienen la independencia de los salarios reales sobre el producto y la "causalidad" del empleo sobre el producto (retroalimentación). En cuanto a la significancia estadistica de los coeficientes, se liene que sólo la suma de los rezagos del producto sobre el empleo son signiticativas al nivel de signilicancia del $5 \%$. El autor realizó un análisis especial para la relación producto-salarios reales para el periodo 1957(2)-1981(2) aplicando variables dummy para el periodo 1976(3)-1978(4) (Drobny,1988,p.192195). Asl se encontró un F-estadi34 relación es inversa).

La razón por la cual dicho efeclo no opera a nivel macro es por el 
efecto ingreso que tiene un cambio de salarios a dicho nivel. Keynes sostiene que un cambio de salarios produce un cambio en el consumo agregado, a través de la redistribución de ingresos que se da entre diversos grupos sociales con diferentes propensiones marginales de consumo. Asl se esperarla que con un incremento en los salarios reales el nivel de consumo real incrementarla, lo cual implica un incremento en el nivel de demanda agregada; por ende, inducirla a un nivel mayor de producción y empleo en la economla. Este efecto podrla ser contrarestado en alguna medida por el cambio en el nivel de inversión. El efecto neto sobre ésta no se puede predecir. Pero con base en el pensamiento de Keynes habrla que analizar como influye un cambio de salarios sobre la demanda por liquidez, como ésta puede afectar a la tasa de interés y de ésta a la inversión. Adicionalmente, en el caso de una economia abierla se puede anticipar un efecto negativo sobre la balanza comercial, ya que el aumento salarial induciria a cierto incremento en el nivel de precios, con lo cual se apreciaria el tipo de cambio real afeclando negalivamenle a las exportaciones y positivamente a las importaciones, lo cual repercute al nivel de producto y empleo de la economia. De ahi que si se desea analizar la consecuencia de cambios generales de los salarios, deberla construirse un modelo macroeconómico que permitiese evaluar las relaciones arriba descritas. ${ }^{10}$

En suma, la idea dominante en el pensamiento Keynesiano es que un aumento on el nivel de gasto real en la economla conlleva a un mayor nivel de producción y empleo cuando las empresas presentan una restricción de demanda. En este marco teórico, las políticas monetarias y fiscales tienen un importante rol para coadyuvar al nivel de la demande efectiva con el fin de mejorar las expectativas tanto de empresarios como de consumidores y asl estimular la inversión y con ello el producto y empleo en las economias.

Los resultados emplricos de las diversas pruebas para examinar si los salarios reales o el nivel de producto es el principal delerminante del empleo agregado no tienen un carácter concluyente todavla.

Lo anterior sigue permitiendo que algunos economistas mantengan posiciones ortodoxas Neoclásicas o Keynesianas, y pueden argumentar que el hecho de no haber encontrado lodavla un insirumento estadistico apropiado para verificar sus hipólesis, no significa que eslas sean erróneas.

Por mi parte, deseo resallar que si bien las diversas pruebas de causalidad-Granger no son los suficientemente consistentes como para ser concluyentes, si han permitido avanzar en ir sugeriendo ciertas tendenclas (las cuales mencionaré mas adelante), que tienen cierta validez, 
en la medida que son denominadores comunes independientemente de la lécnica estadistica especifica que se ha utilizado."

Asl con base en la evidencla emplrica revisada podemos establecer tres importantes tendencias: (a) Es diflcll sostener empirlcamente la existencia de una relación estable entre salarios reales y nivel de empleo; (b) la relaclón producto-empleo se presenta estable en et caso del Reino Unido; tendrla que realizarse pruebas en olros palses y posiblemente realizar pruebas multivariables que Incluyan el nivel de empleo, salarios reales, producto y preclo real de materias primas, ya que estas ullimas han Jugado un papel importante en los 70's; (c) el estudio realizado en el Reino Unido suglere que los salarios reales son independlentes del producto o están positivamente correlacionados, lo cual podría llegar a ser una prueba que el efecto producto efectivamente no opera en la forma indicada por la teorla Neoclásica a nivel macro. No cabe duda, que sigue siendo necesario revisar las actuales metodologlas y/o examinar nuevas, y trabajar con experiencias de diversos palses, en orden a establecer resultados más consistentes que nos permitan ser concluyentes.

Finalmente, es imporlante resaltar que no existe nl en la teorla Neoclásica ni en la Keynesiana (en el aspecto analizado en este trabajo), suficiente y solida corroboración empirica como para sostener postclones ortodoxas, sobre todo, en la aplicación de polílicas económicas. Lastimosamente en los 80's peso mas la ortodoxla Neoclásica que la realidad. Esperamos que en los 90's abandonemos la "ley del péndulo" (pasar de un extremo a otro defendiendo ante lodo posiclones de teo. rias especilicas), y nos volvamos mas abiertos para segulr aprendiendo de la realidad. En este sentido los economistas slempre necesitamos un sano pragmatismo para la adecuación de las teorias a las realidades cambiantes. $^{12}$

\section{Notas}

1. De acuerdo con la deflnición deda por Dombusch en su texto de Macrooconomia, la tasa de desempleo natural es igual a la tase friccional de desempleo que exlste cuando el mercado de trabajo esta en equilibrio. A su voz ol desempleo friccional se da como un resultado del tiempo que lo toma encontrar un nuovo trabajo a los individuos que cambian de trabajo en un momento determinado.

2. Para un análisis mas detallado de la oferta de trabajo puede verse el capitulo 6 del libro texto de Ehrenbero y Smih.

3. Para una exposición mas completa de estos electos puede verse la obra de Drobny p.98-102. Drobny hace una acolaclón sobre el efecto distribución que es la siguiente: Keynes asume que la reducción en los salarios nominales 
conllevara a una reducción de salarios reales tambión. SI ese no fuora of caso, to que ocurrirla dada la mayor proponsión marglnal a consumir de los trabaladores es que a pesar de la calda en los salarios nominales podrla darse un incremento en el nivel de consumo real.

4. Drobny llama a este electo: El efecto Keynes. p.101.

5. Puede verse la propuesta de una melodologla precisa para esto en Haugh (1972).

6. Geary y Kennan calcularon ambas pruebas para los datos on forma diferenciada • indiferenciada. Para el presente trabajo nos limitaremos a analizar al resultado obtonido a través del S-estadistico y con mayor énfasis los resultados que 80 obtuvieron con esta melodologla cuando so aplicaron a los datos y el tamano de la muestra utilizada por Noftcl y Sargent para of caso de los US.

7. Los paises analizados son: Austria, Bélgica, Canadá, Dinamarca, Alemania, Holanda, Irlanda, Halia, Nueva Zelandia, Suiza, Reino Unido y Estados Unidos.

8. So utilizaron siempre datos trimestrales y el deflactor implicito del sector manufacturero.

9. Durante dicho periodo los salarios reales tuvieron un comportamiento bastante variable, el cual se explica mas adelante en este trabajo.

10. Puede verse un modelo construido por Solimano para la economla de Chile. (Solimano,1988).

11. Drobny sehala que la inestabilidad de los resultados según el tamaño muestral que se utilice -entre otros factores - ha sido encontrada también en un estudio hecho por Symons (1985) utilizando el método de ecuaciones estructurales. 